\title{
PROSODIC VARIATION IN CANTONESE-ENGLISH CODE-MIXED SPEECH
}

\author{
Wentao $G u^{1,2}$, Tan Lee ${ }^{1}$ and P. C. Ching ${ }^{1}$ \\ ${ }^{1}$ The Chinese University of Hong Kong, ${ }^{2}$ Nanjing Normal University
}

\begin{abstract}
This study investigates the prosodic features of Cantonese-English code-mixed speech. It is found that the prosody of the matrix language is hardly altered, while the prosody of the embedded language is assimilated to that of the matrix language. That is, the rhythmic pattern is shifted towards syllable-timing, whereas the variations in the $F_{0}$ pattern are mainly in the word-final syllable: for a stressed syllable the $F_{0}$ contour turns flat, while for a posttonic unstressed syllable the $F_{0}$ contour falls more steeply than in monolingual English speech. Such $F_{0}$ variations can be explained by the phonological interaction of English lexical stress and Cantonese lexical tone. In addition, the $F_{0}$ of the embedded English word tends to become higher due to the embedding effect.
\end{abstract}

Index Terms - Code-mixing, timing, $F_{0}$, stress, tone

\section{INTRODUCTION}

In linguistics, code-switching/mixing refers to using more than one language or variety in conversation. It is a common phenomenon in multilingual societies, along with other language contact phenomena such as borrowing (loanword), calques, pidgins and creoles, and language interference. Usually two terms are used to differentiate the phenomenon at two different levels: codeswitching mainly refers to the switches between clauses or sentences (inter-sentential), while code-mixing refers to the switches within a single clause or sentence (intra-sentential).

Hong Kong is a well-known multilingual society, and codeswitching/mixing is deeply rooted in the everyday life of Hong Kong residents, especially in casual conversation [1]. The switches are mostly between Cantonese and English since the majority of Hong Kong residents are Cantonese-English bilinguals, though for most of them the only native language is Cantonese. Such switches usually occur intrasententially, hence referred to as code-mixing. The syntax of the sentence follows Cantonese grammar and applies to the English words (including compounds and phrases) that are embedded in the sentence - called 'code-switch words'. Thus, Cantonese is the primary or matrix language, while English is the secondary or embedded language.

In recent years, code-switching/mixing has received more attention. On the one hand, it is no longer a peripheral topic in the narrow tradition of research on bilingualism but has attracted more general interests of sociolinguists, psycholinguists and general linguists, especially on sociocultural meaning and syntactic constraints. On the other hand, the technologies for processing code-mixed speech have also been developed, e.g., automatic speech recognition (ASR) [2], which is more challenging for codemixed speech than for monolingual speech as well as multilingual speech with the language boundaries known in advance.

In contrast, the phonetic and phonological investigation of the pronunciation variation caused specifically by code-mixing is rather limited. Only very few studies discussed the segmental variation in terms of human speech recognition [3] or the phone recognition results by ASR algorithm [4] - though still lacking a further acoustic-phonetic analysis in both cases. The reports on the suprasegmental variation in code-mixed speech are even fewer. A prosodic study on Mandarin-English code-mixed speech [5] showed that the prosodic features of the stretch of speech of the matrix language (Mandarin) are hardly altered, while the stretch of speech of the embedded language (English) exhibits a higher $F_{0}$ and a steeper slope of $F_{0}$ falling than in monolingual English speech. However, the statistical results there are crude, and there still lacks a convincing phonetic and phonological interpretation.

It is quite likely that the suprasegmental variation in codemixing is more prominent than the segmental variation, because the suprasegmental interaction is by definition more compulsive (as suprasegmental features span a wider stretch of speech), even for those who speak the embedded language as fluently as natives. Such interaction deserves in-depth study, especially when the two languages are quite different in prosodic phonology, which is the case for Cantonese-English code-mixing. In the present study we conduct a preliminary investigation into the timing and $F_{0}$ patterns.

\section{PHONOLOGIES OF THE TWO LANGUAGES AND THEIR INTERACTION}

\subsection{Phonologies of Cantonese and English}

The two languages, Cantonese and English, contrast sharply in phonological structure.

Cantonese, as one of the major Chinese dialects, is a tone language of monosyllabic nature in the morpheme. It has a very simple syllable structure $(\mathrm{C}) \mathrm{V}(\mathrm{C})$, where $\mathrm{V}$ is a vowel and $\mathrm{C}$ is an optional consonant. Cantonese syllables have six lexical tones (the entering tones in checked syllables can be merged with their nonentering counterparts due to similar pitch patterns), as listed below:

$\begin{array}{lll}\text { T1 } & \text { high level } & 55 \\ \text { T2 } & \text { high rising } & 25 \\ \text { T3 } & \text { mid level } & 33 \\ \text { T4 } & \text { low falling } & 21 \\ \text { T5 } & \text { low rising } & 23 \\ \text { T6 } & \text { low level } & 22\end{array}$

The third column gives the 5-level tone-letter notation - the levels 1 to 5 , indicate relative pitch values from low to high, respectively. Cantonese does not have lexical stress, nor a neutral tone functioning as unstress. Also, Cantonese is a typical syllable-timed language, for which each syllable in connected speech is perceived as taking roughly a constant amount of time. Namely, the rhythm tends very roughly to have a syllable-based isochrony.

English, on the contrary, has a more complex syllable structure than Cantonese: by adopting consonant clusters, the syllables have the structure of $(C)(C)(C) V(C)(C)(C)(C)$. English is a stress language, for which the syllables in a word differ in the 
degree of lexical stress that signals the perceived salience (in English usually three degrees of lexical stress are distinguished: primary stress, secondary stress, and unstress), and each word has at least one stressed syllable in its citation form. Also, English is a typical stress-timed language, for which a roughly constant amount of time is perceived between consecutive stressed syllables in connected speech (they are generally the syllables being lexically stressed in words, but not vice versa). Namely, the rhythm tends very roughly to have a stress-based isochrony. To accommodate this, unstressed syllables in the utterance are comparatively shorter.

\subsection{Interaction in prosodic phonology}

In code-mixed speech, the pronunciation of the code-switch words tends to be assimilated to that of the matrix language to a certain extent. As discussed in $[1,4]$, there are two types of segmental variation for the English words in Cantonese-English code-mixed speech: (1) The syllable structure of English words is sometimes modified into that of Cantonese, by means of phone insertion and phone deletion; (2) Phone change: the phones unique to English may be pronounced as similar phones in Cantonese.

For suprasegmental features, it is recognized that lexical stress in stress languages can be reinterpreted as tonal contrast when embedded in tone languages. A well-known example given by Cheng [6] showed explicitly that Mandarin treats English stress and unstress as high tone $(\mathrm{H}$; or 55$)$ and low tone $(\mathrm{L} ;$ or 21$)$ :

hao3 pro'fessor (good professor) $\rightarrow$ hao 2 pro'fessor L L H L LH L H L

hao3 'student (good student)

L H L

Here the T3 sandhi $(\mathrm{L} \rightarrow \mathrm{LH} / \mathrm{L}$, where $\mathrm{LH}$ and $\mathrm{L}$ denote the pitch patterns of T2 and T3 of Mandarin, respectively) on /hao3/ is triggered only when the following English syllable is unstressed.

Such a relationship between stress and tone is also seen in the tonal patterns of Cantonese loanwords of English origin [7]. The rules proposed in [7] can be approximately summarized as follows: (1) English stressed syllables (in primary or secondary stress) carry a high tone 55 (T1).

(2) Pre-tonic unstressed syllables carry a low tone 22 (T6), if epenthetic; otherwise a mid tone 33 (T3)

(3) Post-tonic unstressed syllables carry a low-falling tone 21 (T4) or a high-rising tone 25 (T2). The former is usually produced by those who are good at English pronunciation, while the latter sounds more like colloquial Cantonese, in which a lexically nonhigh tone in the word-final syllable is often changed into T2.

Although loanword and code-mixing are language contact phenomena at two different levels (loanword occurs within the lexicon, while code-mixing occurs at the syntactic level), they are quite likely to be similar in adapting the prosodic pattern of the embedded language towards that of the matrix language. The aforementioned interaction between English lexical stress and Cantonese lexical tone is expected to exist in code-mixing as well.

\section{SPEECH DATA}

It is known that the prosodic features such as duration and $F_{0}$ vary with a number of contextual factors. To investigate the variation caused specifically by code-mixing, we designed a controlled experiment to compare the prosody of a given stretch of speech occurring both in monolingual and in code-mixed utterances.

Since the position of lexical stress in English word may play an important role, we differentiated five different types of English
Table 1. English words embedded in Cantonese sentences.

\begin{tabular}{cc}
\hline \hline Type of word & Word list \\
\hline Monosyllabic word & pass; pub \\
\hline $\begin{array}{c}\text { Disyllabic word with lexical stress } \\
\text { on the first syllable }\end{array}$ & 'bonus; 'median \\
\hline $\begin{array}{c}\text { Disyllabic word with lexical stress } \\
\text { on the second syllable }\end{array}$ & can'teen; re'view \\
\hline $\begin{array}{c}\text { Word with more than two syllables } \\
\text { Complex word } \\
\text { (compound or phrase) }\end{array}$ & a'ssignment; , corre'lation \\
\hline \hline
\end{tabular}

words as listed in Table 1, where the lexical stresses (of two degrees) in polysyllabic words are marked (monosyllabic words have a default stress, though unmarked; for complex words, the lexical stresses in all element words are marked). For the sake of comparison, all the words selected here, when embedded in Cantonese utterances, are subject neither to any change in syllable number, nor to the colloquial word-final $\mathrm{T} 2$ change which occurs for some post-tonic unstressed syllables, as found in loanwords.

All the listed 11 English words can be embedded very naturally in Cantonese utterances. For each of them, nine sentences were designed, corresponding to the combination of three language conditions (monolingual Cantonese, monolingual English, and Cantonese-English code-mixing; henceforth denoted as C, E, and $\mathrm{CE}$, respectively) and three sentential positions for the target words (sentence-initial, medial, and final). Each set of three sentences in different language conditions have the same meaning and the same sentential position of the target word (though the exact sententialmedial position may differ due to different sentence lengths). For example, the following lists a set of three sentences with the target word "correlation (關係)" embedded in the mid of the sentence:

\section{$\mathrm{CE}$ : 我都唔知原來個 correlation 咁大。}

$\mathrm{C}$ : 我都唔知原來個 關係 咁大。

E: I don't even know the correlation is so high.

Since code-mixing occurs usually in casual conversation, the Cantonese text is given in colloquial style instead of formal written style. All the sentences are declarative, hence inherently associated with a declining intonation.

The informants are four native speakers of HK Cantonese, including two males and two females, who are all graduate students. They speak English fluently but not at the native level, i.e., still with HK accent. The HK accent in their monolingual English utterances, however, is not a problem here, because the present study is only concerned with the variation caused by codemixing, by taking their monolingual English speech as a reference.

Each text sentence was recorded with three repetitions at the normal speech rate of each speaker. Hence there are altogether 11 $\times 9 \times 3=297$ utterances for each speaker. Due to space limitations, only the results for the two female speakers (FA and FB) will be presented, though similar results were also found for the two males.

The utterances were segmented manually into syllables by visual inspection of waveform and spectrogram. For each utterance, the raw $F_{0}$ values extracted by an autocorrelation analysis were smoothed and interpolated to produce a continuous $F_{0}$ contour, which was then time-normalized by extracting ten $F_{0}$ points at equal time interval in each syllable. The average time-normalized $F_{0}$ contour for each sentence was obtained by averaging over the three repetitions of utterances. Also, before time-normalization, a linear regression was conducted on the continuous $F_{0}$ contour in each syllable to estimate the best-approximated syllabic $F_{0}$ slope. 
Table 2. Durational proportion of the primarily stressed syllable in the entire English word for FA (in \%).

\begin{tabular}{ccccccc}
\hline \multirow{2}{*}{ Word } & \multicolumn{2}{c}{ Initial } & \multicolumn{2}{c}{ Medial } & \multicolumn{2}{c}{ Final } \\
\cline { 2 - 7 } & $\mathrm{CE}$ & $\mathrm{E}$ & $\mathrm{CE}$ & $\mathrm{E}$ & $\mathrm{CE}$ & $\mathrm{E}$ \\
\hline 'bonus & 38.7 & 38.7 & 47.8 & 55.6 & 41.5 & 46.7 \\
'median & 35.7 & 39.2 & 43.3 & 49.1 & 44.6 & 48.9 \\
can'teen & 51.7 & 48.1 & 47.9 & 48.3 & 54.7 & 54.8 \\
re'view & 53.8 & 55.9 & 50.9 & 68.5 & 61.7 & 67.6 \\
a'ssignment & 50.5 & 52.4 & 45.0 & 55.1 & 48.1 & 53.7 \\
'corre,lation & 28.0 & 28.6 & 35.1 & 37.1 & 36.7 & 36.7 \\
,annual 'leave & 35.9 & 41.3 & 34.1 & 37.4 & 46.4 & 46.5 \\
'grant ,loan & 40.2 & 49.2 & 54.1 & 59.2 & 46.7 & 54.1 \\
'student ,union & 37.9 & 36.3 & 28.3 & 29.3 & 28.1 & 32.5 \\
\hline Average & 41.4 & 43.3 & 42.9 & 48.8 & 45.4 & 49.1 \\
\hline \hline
\end{tabular}

Table 3. Durational proportion of the primarily stressed syllable in the entire English word for FB (in \%).

\begin{tabular}{ccccccc}
\hline \hline \multirow{2}{*}{ Word } & \multicolumn{2}{c}{ Initial } & \multicolumn{2}{c}{ Medial } & \multicolumn{2}{c}{ Final } \\
\cline { 2 - 7 } & $\mathrm{CE}$ & $\mathrm{E}$ & $\mathrm{CE}$ & $\mathrm{E}$ & $\mathrm{CE}$ & $\mathrm{E}$ \\
\hline 'bonus & 31.2 & 34.1 & 42.4 & 47.6 & 33.1 & 33.4 \\
re'view & 72.3 & 77.8 & 62.4 & 71.9 & 72.3 & 76.2 \\
a'ssignment & 47.4 & 50.9 & 46.4 & 48.2 & 41.1 & 42.8 \\
'grant, loan & 40.2 & 49.2 & 54.1 & 59.2 & 46.7 & 54.1 \\
$\ldots \ldots$ & $\ldots \ldots$ & $\ldots \ldots$ & $\ldots \ldots$ & $\ldots \ldots$ & $\ldots \ldots$ & $\ldots \ldots$ \\
\hline Average & 41.3 & 44.6 & 42.5 & 46.4 & 42.6 & 44.8 \\
\hline \hline
\end{tabular}

\section{VARIATION IN TIMING PATTERN}

In the first place, the English words shared by the code-mixed utterances and the monolingual English utterances were compared. For the duration of the entire target word, no significant and consistent difference was observed. We then further examined the intra-word durational proportion of the syllables within the word.

Other things being equal, a stressed syllable is generally longer than an unstressed syllable in a language that distinguishes stress and unstress. Moreover, as English is a stress-timed language, unstressed syllables tend to be further shortened. On the contrary, since Cantonese makes no lexical distinction of stress vs. unstress and is also a syllable-timed language, syllable duration in Cantonese tends to be less variable.

To examine the rhythmic interaction in code-mixing, the durational proportion of the primarily stressed syllable in the polysyllabic English target words were calculated. The results are shown in Tables 2 and 3, for the two speakers, respectively. Due to space limitations, only the results for some words and the average result are given in Table 3. The primarily stressed syllables are marked in bold. For a complex word (compound or phrase), the lexical stresses in two element words are modified in the way that one is given the primary stress while the other is given the secondary. However, it is not always clear to tell which element word is stressed more, especially for non-native speakers. Hence, the primary stresses here are marked according to the speakers' actual pronunciation in monolingual English utterances, though the pronunciation may not be correct. This criterion is also applied to the word "correlation", for which FA placed the primary stress incorrectly on the first syllable - we mark it as it was pronounced.

For most target words, the intra-word durational proportion of the primarily stressed syllable in code-mixed speech is smaller than in monolingual English speech. This indicates that the rhythm of the embedded English words is shifted towards syllable-timing, i.e., assimilated to the rhythm of Cantonese, the matrix language. In

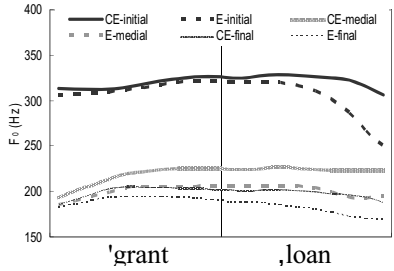

(a) Speaker FA

Figure 1: Time-normalized $F_{0}$ contours of the word "grant loan".

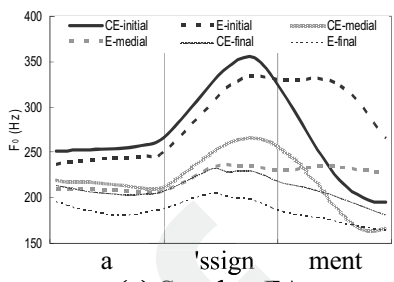

(a) Speaker FA

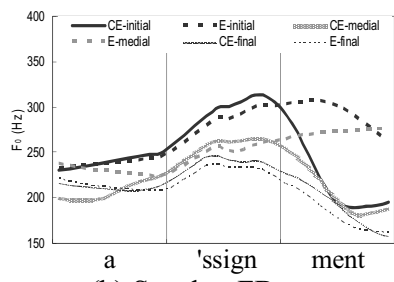

(b) Speaker FB

Figure 2: Time-normalized $F_{0}$ contours of the word "assignment".

comparison, the decrease in durational proportion is the most conspicuous at the sentence-medial position, probably because the word embedded in the mid of the sentence is confined by the matrix language from both sides and hence is assimilated the most.

Next, we also compared the Cantonese part shared by the CEutterances and the C-utterances. However, no significant and consistent difference was observed, neither for the duration of the entire stretch of speech (i.e., the tempo) nor for the duration of the boundary syllables next to the embedded target word. It indicates that the timing pattern of the matrix language remains intact.

\section{VARIATION IN $\boldsymbol{F}_{\mathbf{0}}$ PATTERN}

Ignoring durational difference, the syllable-based time-normalized $F_{0}$ contours of the English target words embedded in six types of sentences (CE and E, at each of the three sentential positions) can be aligned for display and comparison. For example, Figs. 1 and 2 show the average time-normalized $F_{0}$ contours for the words "grant loan" and "assignment" in all the six conditions.

In both figures, the overall $F_{0}$ height, except in the ending syllable of "assignment", ranks largely in a consistent order: CEinitial $>$ E-initial $>$ CE-medial $>$ E-medial $>$ CE-final $>$ E-final. The difference among the three sentential positions, especially evident between initial and non-initial positions, reflects the declination effect. At each sentential position, the $F_{0}$ of the English target word in CE-utterances is usually higher than in E-utterances. Thus, like also reported in [5], $F_{0}$ raising is a manifestation of the embedding effect, which triggers some degree of prominence. The pattern is also observed in other samples, which can be explicitly seen from the mean $F_{0}$ 's for all target words (except the post-tonic unstressed syllables) as listed in Table 4 . The $F_{0}$ raising due to the embedding effect is most conspicuous in the mid of the sentence.

In the above, we did not compare $F_{0}$ height in the word-final syllable "ment", because the $F_{0}$ contour of the word-final syllable may vary greatly, not only in the height but also in the shape. The two figures show exactly two opposite patterns. The final syllable of the word "grant loan" is stressed (though in secondary stress), and the $F_{0}$ contour of this syllable in the CE-utterances is usually flatter than in the E-utterances. In contrast, the final syllable of the word "assignment" is post-tonic unstressed, and the $F_{0}$ contour of 
this syllable in the CE-utterances usually gives a much steeper falling trajectory than in the E-utterances, though less evident at the sentential-final position due to the final-lowering effect.

A more accurate comparison of $F_{0}$ slopes is provided by the results of linear regression on the syllabic $F_{0}$ contours before timenormalization. Table 5 gives the best-estimated $F_{0}$ slopes in the ending syllables of all the English target words for FA. The words are grouped into two categories in terms of the ending stress condition (stressed vs. post-tonic unstressed). They exhibit exactly the two opposite patterns as shown above in Figs. 1 and 2. Due to space limitations, for FB only the average values are given in Table 6. In addition, the non-word-final syllables also exhibit a similar pattern, as shown in Table 6. That is, in CE-utterances, the rising $F_{0}$ in a stressed syllable rises more steeply (as is also seen in Figs. $1-2$ ), while the $F_{0}$ in a post-tonic unstressed syllable tends to fall more steeply, though such differences are less evident than in the case of word-final syllable.

Such phenomena can be explained by the interaction between English lexical stress and Cantonese lexical tone - stressed and post-tonic unstressed syllables of English are encoded, respectively, to high (55) and low (21) tones, as discussed in $[6,7]$. In codemixed speech, if the ending syllable of an English word is stressed, it is encoded to a high-level tone, which gives a flat $F_{0}$ contour instead of an $F_{0}$ contour with a falling end in monolingual English speech. Otherwise, if the ending syllable is post-tonic unstressed, it is encoded to a post-H low-falling tone, which gives a much steeper falling contour than in monolingual English utterances.

All these $F_{0}$ variations are ascribed to the different natures in pitch for the two languages: in contrast to English, Cantonese $F_{0}$ contours on the one hand can have very flat local sections for level tones, and on the other hand the local rises and falls can be even steeper for characterizing contour tones and tonal transitions. In a word, tone languages have a finer control on local tonal targets.

Next, the time-normalized $F_{0}$ contours of the Cantonese part in six types of sentences ( $\mathrm{CE}$ and $\mathrm{C}$, with target words embedded at each of the three sentential positions) were also compared. The difference is not significant (either in $F_{0}$ height or in $F_{0}$ shape), indicating that the $F_{0}$ of the matrix language is hardly altered.

\section{CONCLUSION}

This study investigated the prosodic features of Cantonese-English code-mixed speech. Due to the embedding effect, the $F_{0}$ level of the code-switch word tends to be raised. More importantly, there is a tendency that the prosody of the embedded language is assimilated to that of the matrix language. The rhythmic pattern is shifted towards syllable-timing, while the variations in $F_{0}$ pattern are mainly in the word-final syllable: for a stressed syllable the $F_{0}$ contour turns flat, while for a post-tonic unstressed syllable the $F_{0}$ contour falls more steeply. Such $F_{0}$ variations are the result of the phonological interaction of English lexical stress and Cantonese lexical tone. In contrast, the prosody of the matrix language is hardly altered. These discoveries in prosodic features are expected to be useful for developing ASR or TTS systems for code-mixed speech.

\section{ACKNOWLEDGEMENT}

This work is supported partly by a grant awarded by Shun Hing Institute of Advanced Engineering, The Chinese University of Hong Kong, and an Earmarked Grant (Ref: CUHK 411306) by the Hong Kong Research Grants Council.
Table 4. Mean $F_{0}$ 's of the embedded English words except the post-tonic unstressed word-final syllables (in $\mathrm{Hz}$ ).

\begin{tabular}{ccccccc}
\hline \hline \multirow{2}{*}{ Speaker } & \multicolumn{2}{c}{ Initial } & \multicolumn{2}{c}{ Medial } & \multicolumn{2}{c}{ Final } \\
\cline { 2 - 7 } & CE & E & CE & E & CE & E \\
\hline FA & 310 & 302 & 254 & 235 & 222 & 210 \\
FB & 296 & 288 & 256 & 243 & 230 & 220 \\
\hline \hline
\end{tabular}

Table 5. $F_{0}$ slopes in the ending syllables of the embedded English words for $\mathrm{FA}$ (in $\mathrm{Hz} / \mathrm{s}$ ).

\begin{tabular}{ccccccc}
\hline \hline \multirow{2}{*}{ Word } & \multicolumn{2}{c}{ Initial } & \multicolumn{2}{c}{ Medial } & \multicolumn{2}{c}{ Final } \\
\cline { 2 - 7 } & $\mathrm{CE}$ & $\mathrm{E}$ & $\mathrm{CE}$ & $\mathrm{E}$ & $\mathrm{CE}$ & $\mathrm{E}$ \\
\hline pass & -84 & -247 & 71 & 85 & -22 & 38 \\
pub & -82 & -512 & 215 & 189 & -57 & -106 \\
can'teen & 204 & 160 & 94 & 11 & 39 & -99 \\
re'view & 512 & 512 & 236 & 142 & 98 & -32 \\
,annual 'leave & -202 & -272 & -80 & -85 & -68 & -145 \\
'grant ,loan & -74 & -397 & -15 & -113 & -55 & -117 \\
\hline Average & 46 & -126 & 87 & 38 & -11 & -77 \\
\hline 'bonus & -560 & -486 & -467 & -30 & -329 & -388 \\
'median & -468 & -461 & -378 & -144 & -387 & -289 \\
a'ssignment & -884 & -416 & -671 & -28 & -524 & -323 \\
'corre,lation & -709 & -347 & -408 & -246 & -342 & -320 \\
'student, union & -710 & -664 & -518 & -474 & -642 & -454 \\
\hline---656 & -475 & -488 & -185 & -445 & -355 \\
\hline Average & -666 & -475 &
\end{tabular}

Table 6. Average $F_{0}$ slopes in the syllables of the embedded English words for $\mathrm{FB}$ (in $\mathrm{Hz} / \mathrm{s}$ ).

\begin{tabular}{cccccccc}
\hline \hline \multirow{2}{*}{ Type of syllable } & \multicolumn{3}{c}{ Initial } & \multicolumn{2}{c}{ Medial } & \multicolumn{2}{c}{ Final } \\
\cline { 2 - 8 } & CE & E & CE & E & CE & E \\
\hline \multirow{2}{*}{ word-ending post-tonic unstrs. } & -528 & -122 & -467 & -58 & -542 & -444 \\
\hline \multirow{2}{*}{ non-ending } & stressed & 177 & 138 & 193 & 116 & 112 & 29 \\
& post-tonic unstrs. & -40 & -53 & -66 & 51 & -86 & -60 \\
\hline \hline
\end{tabular}

\section{REFERENCES}

[1] Chan, B. H. S., "Code-mixing in Hong Kong CantoneseEnglish bilinguals: Constrains and processes," MA thesis, The Chinese University of Hong Kong, 1992.

[2] Chan, J. Y. C., Ching, P. C., Lee, T., and Cao, H., "Automatic speech recognition of Cantonese-English code-mixing utterances," Proc. Interspeech 2006, pp. 113-116, Pennsylvania, USA, 2006.

[3] Li, P., "Spoken word recognition of code-switched words by Chinese-English bilinguals," Journal of Memory and Language, vol. 35, pp. 757-774, 1996.

[4] Cao, H., Lee, T., and Ching, P. C. "A study of pronunciation variation in Cantonese-English code-mixing speech," Proc. Oriental COCOSDA 2007, pp. 143-148, Hanoi, Vietnam, 2007.

[5] Chen, G. P., "Prosody modeling in multilingual speech synthesis," MA thesis, University of Science and Technology of China, 2005.

[6] Cheng, C., "English stresses and Chinese tones in Chinese sentences," Phonetica, vol. 18, pp. 77-88, 1968.

[7] 张日昇, “香港广州话英语音译借词的声调规律” , 中国 语文，第 1 期，42-50，1986。 